\title{
Correction to: A New Extended VIKOR Approach Using q-Rung Orthopair Fuzzy Sets for Sustainable Enterprise Risk Management Assessment in Manufacturing Small and Medium- Sized Enterprises
}

\author{
Sun Cheng ${ }^{1} \cdot$ Sun Jianfu $^{1} \cdot$ Melfi Alrasheedi $^{2} \cdot$ Parvaneh Saeidi $^{3} \cdot$ Arunodaya Raj Mishra $^{4} \cdot$ \\ Pratibha Rani ${ }^{5}$
}

Published online: 28 October 2021

(C) Taiwan Fuzzy Systems Association 2021

Correction to: Int J Fuzzy Syst (2021)

https://doi.org/10.1007/s40815-020-01024-3

In the published article [1], the affiliations 2 and 3 were published incorrectly. They should be corrected as follows.

${ }^{2}$ Department of Quantitative Methods, School of Business, King Faisal University, Al-Ahsa, Saudi Arabia

${ }^{3}$ Facultad de Ciencias Adminsitrativas y Económicas, Universidad Tecnológica Indoamérica, Quito, Ecuador

The authors apologize for any inconvenience that it may have caused.

\section{Reference}

1. S Cheng S Jianfu M Alrasheedi 2021 A new extended VIKOR approach using q-rung orthopair fuzzy sets for sustainable enterprise risk management assessment in manufacturing small and medium-sized enterprises Int. J. Fuzzy Syst. 2313471369 https://doi.org/10.1007/s40815-020-01024-3
The original article can be found online at https://doi.org/10.1007/ s40815-020-01024-3.

Sun Jianfu

sjianfu@163.com

1 School of Management, Hebei University,

Baoding 071002, Hebei Province, China

2 Department of Quantitative Methods, School of Business, King Faisal University, Al-Ahsa, Saudi Arabia

3 Facultad de Ciencias Adminsitrativas y Económicas, Universidad Tecnológica Indoamérica, Quito, Ecuador

4 Department of Mathematics, Govt. College Jaitwara, Satna, MP, India

5 Department of Mathematics, National Institute of Technology, Warangal 506004, India 